\title{
THE NATURE OF SUPERCONDUCTING FLUCTUATIONS AND NEW ASPECTS IN HIGH TC SUPERCONDUCTORS
}

\author{
ALESSANDRO LASCIALFARI $(* * * *)$ e ATTILIO RIGAMONTI $(* *)$
}

Nota presentata dal Prof. A. Lascialfari

(Adunanza del 29 giugno 2017)

\begin{abstract}
SUNTO. - Dopo una breve introduzione sulle fluttuazioni superconduttive, si presentano tipici risultati sperimentali in diversi sistemi. Le fluttuazioni superconduttive sono note esistere sin dagli anni '60 e sono state evidenziate dapprima in nanoparticelle di superconduttori convenzionali. Nelle ultime decadi, attraverso misure di diamagnetismo precursore al di sopra della temperatura critica $T_{c}$, sono stati messi in evidenza particolari aspetti delle fluttazioni nei superconduttori ad alta $\mathrm{T}_{c}$, particolarmente in quelli definiti "sotto-drogati". I risultati ottenuti, qui riassunti, sono stati interpretati in termini di fluttuazioni della fase del parametro d'ordine superconduttivo, una funzione complessa. Un modello teorico, già sviluppato per condizioni rigorose di dimensionalità nulla (quindi in sistemi di nanoparticelle) è stato da noi esteso a includere approssimativamente anche sistemi a dimensioni finite, dell'ordine della distanza di coerenza tra isole locali di superconduttività fluttuante. Sono cosi stati descritti e giustificati i risultati di diamagnetismo fluttuante in cuprati, pnictidi e anche in superconduttori a base Fe. Oltre a questi risultati, qui riassunti, vengono anche presentati risultati preliminari ottenuti in superconduttori a base $\mathrm{Ca}$. Si mostra come un particolare parametro (il campo di upturn) ove la magnetizzazione diamagnetica inizia a diminuire al crescere del campo, consente di discriminare con sicurezza gli effetti di natura squisitamente superconduttiva da similari effetti di natura più convenzionale, legati a una distribuzione di $\mathrm{T}_{\mathrm{c}}$ per disomogeneità di carattere chimico.
\end{abstract}

(*) Dipartimento di Fisica \& INSTM, Università degli Studi di Pavia, Italy.

E-mail: alessandro.lascialfari@unipv.it

(**) Dipartimento di Fisica "A. Volta", Università degli Studi di Pavia, Italy.

E-mail: attilio.rigamonti@unipv.it 
$* * *$

ABSTRACT. - After a brief introduction to thermodynamical superconducting fluctuations, some experimental results in different systems are presented. Superconducting fluctuations were predicted and revealed in nanoparticles of conventional superconductors since late 60s. However, just in the last two decades phenomena related to an anomalous diamagnetism have been discovered in high- $\mathrm{T}_{c}$ underdoped compounds, mainly by collecting the isothermal magnetization curves above the critical temperature $\mathrm{T}_{\mathrm{c}}$. These experimental data can be interpreted in terms of fluctuations of the phase of the order parameter and a related theoretical model can explain the results in cuprates, pnictides and Fe-based materials. Such results, here presented together with preliminary data on a Ca-based high $-\mathrm{T}_{\mathrm{c}}$ system, allow one to extract a fundamental parameter, the upturn magnetic field above which the diamagnetic magnetization initiates to decrease on increasing the field. Its temperature dependence distinguishes among diamagnetic effects related to superconducting phase fluctuations and to analogous effects due to chemical inhomogenities.

\section{FLUCTUATIONS, SUPERCONDUCTING FLUCTUATIONS AND RELATED DIAMAGNETISM}

The concept of fluctuations of a specific quantity, is spreaded across different disciplines, ranging from e.g. economy, finance, health sciences, mathematics, chemistry and physics. In physics, one of well known experimental evidences of fluctuations (of thermodynamical type), is the density fluctuations in liquids, generating the critical opalescence effect, observed since centuries ago, that can be described by means of the Ginzburg-Landau (GL) theory. As a translation of the GL theory of fluctuations in correspondence of the transition critical temperature in different systems (magnetic, superconducting, superflu$\mathrm{id}$, etc), superconducting fluctuations (SF) of the order parameter (the Cooper pair wave-function $[1,2]$ ) were evidenced by measurements mainly of the resistivity, the magnetization and the susceptibility just above $\mathrm{T}_{\mathrm{c}}$ [3]. After the discovery of high- $\mathrm{T}_{\mathrm{c}}$ systems [4], through STM microscope it was possible to observe directly superconducting fluctuations in the system $\mathrm{La}_{2-\mathrm{x}} \mathrm{Sr}_{\mathrm{x}} \mathrm{CuO}_{4}$.

From the quantitative point of view, the order parameter of superconductors is represented by the wave-function of the Cooper pairs: $\Psi=|\Psi| \mathrm{e}^{\mathrm{i}}$, where $|\Psi|$ is the modulus and $\vartheta$ is the phase [5]. The square modulus of this paramater can be locally different from zero for $\mathrm{T} \geq \mathrm{T}_{c}$, where Cooper pairs start to form in metastable states: the system is preparing for the transition to the superconducting phase (Fig. 1). 


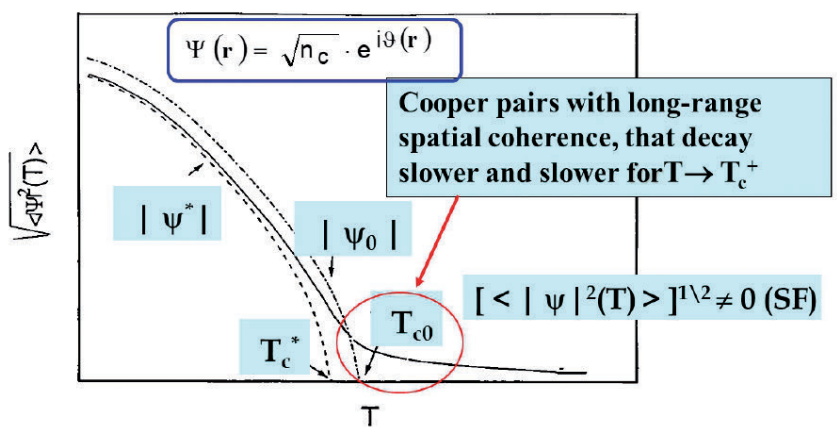

Fig. 1 - The order parameter is renormalized by the fluctuations. Dashed line: curve deduced from the Bardeen-Cooper-Schrieffer theory, with $T_{c}^{*}$ renormalized by fluctuations. Continuous line: T-dependence of $|\Psi|$ renormalized by fluctuations.

The occurrence of superconducting fluctuations has relevant consequences: the "fluctuating" Cooper pairs above $\mathrm{T}_{\mathrm{c}}$ imply the presence of (fluctuating) diamagnetism and of excess conductivity (Fig. 2). A qualitative understanding of the fluctuating diamagnetism (FD) can be achieved by considering the two electrons (of the Cooper pairs) at an average distance of the order of $\xi_{\mathrm{GL}}(\mathrm{T})$ (the Ginzburg-Landau coherence length).
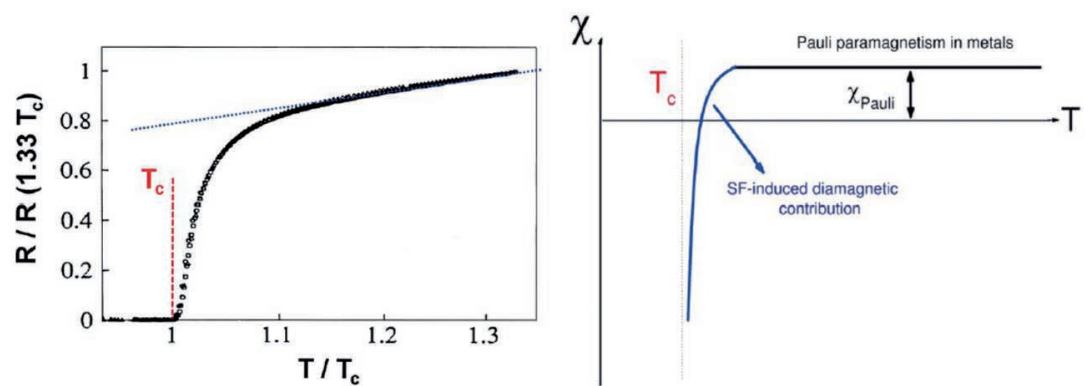

Fig. 2 - Left: renormalized eletrical resistance as a function of reduced temperature. Above $T_{c}$ where the SF set in, the resistance is decreased with respect to metallic behaviour (blue line). Right: effect of magnetic susceptibility "smoothing" just above $T_{0}$ as consequence of the fluctuating diamagnetism.

The theory of FD in conventional superconductors (SC) in the limit of zero field has an exact solution, proposed by Prange $[2,5,6]$, but no field quenching of fluctuating pairs is taken into account. In the 
1960s, Gollub, Tinkham et al $[7,8]$, demonstrated experimentally in metals the breakdown of a description which neglects the effect of the field in suppressing the fluctuating pairs. Further developments of the GL theory of SF in the gaussian approximation, allowed to extract the H-dependence of the magnetization (Fig. 3).

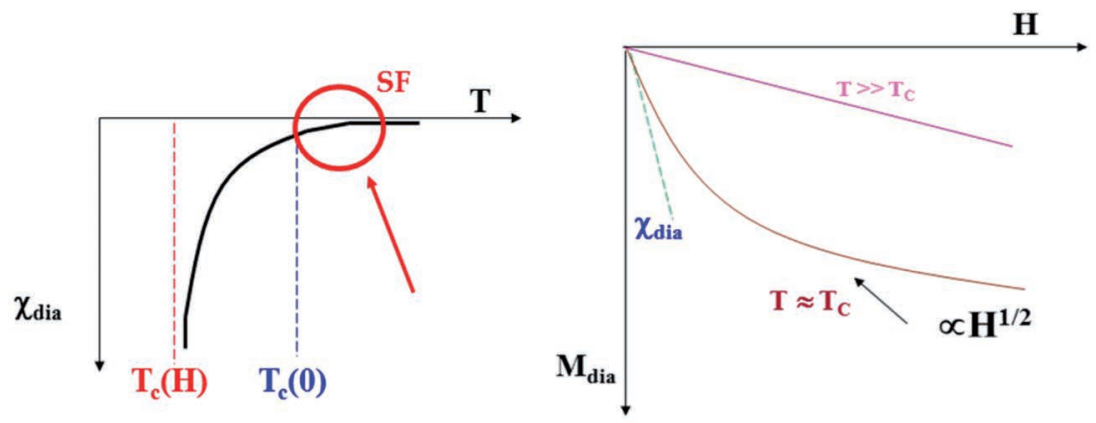

Fig. 3 - Sketchy T-behaviour of magnetic susceptibility (after subtraction of the Pauli term) and H-behaviour of the isothermal magnetization curves in the presence of SF. The curves on the right of the figure report the behaviour according to the GL theory in the gaussian approximation.

\section{Superconducting Fluctuations in Pb NANOPARTICles: THE ZERO-DIMENSIONAL CASE}

A case where the theory of superconducting fluctuations has been directly verified, is the zero- $\mathrm{D}(0 \mathrm{D})$ case for $\mathrm{Pb}$ nanoparticles [2,5]. In Fig. 4 the diamagnetic magnetization $\mathrm{M}_{\text {dia }}$ is reported as a function of $\mathrm{H}$ for 3 distinct temperatures just above $T_{c}\left(T_{c}(0)=7.09 \mathrm{~K}\right)$, in the sample with average diameter $d=750 \AA$. The solid lines represent the best fit obtained by using the $0 \mathrm{D}$ equations [5], derived from complete GL theory in $0 \mathrm{D}$ (i.e. by including the $\left|\Psi_{0}\right|^{4}$ term in the free energy expression $[2,5])$. It is noted the presence of an experimental "upturn field" $\mathrm{H}_{\text {up }}$ that shifts toward lower fields as the temperature is decreased: $\mathrm{H}_{\text {up }}$ $=110,67,60$ Oe. The zero-dimensional condition is applied to the evanescent SC droplets of size which causes the maximum diamagnetic screening. The magnetic susceptibility data present clearly the diamagnetic effect, reported in Fig. 5 for two different particles dimensions. In the $0 \mathrm{D}$-case it could be noted that, by means of GL theory, a direct eval- 
uation of the thermodynamical free energy can be made and the effect of $\mathrm{H}$ can be exactly taken into account [9].

Interestingly the data in $\mathrm{MgB}_{2}$ are qualitatively explained for $\xi(\mathrm{T} \rightarrow 0) \approx 100 \AA$ and $10^{-3}<\varepsilon<10^{-2}\left(\varepsilon=\left(\mathrm{T}-\mathrm{T}_{\mathrm{c}}\right) / \mathrm{T}_{\mathrm{c}}\right)$; in this case one obtains $\mathrm{H}_{\text {up }} \approx 100 \div 1000$ Oe by using the expression of diamagnetic magnetization in the $0 \mathrm{D}$ case $[10]$ :

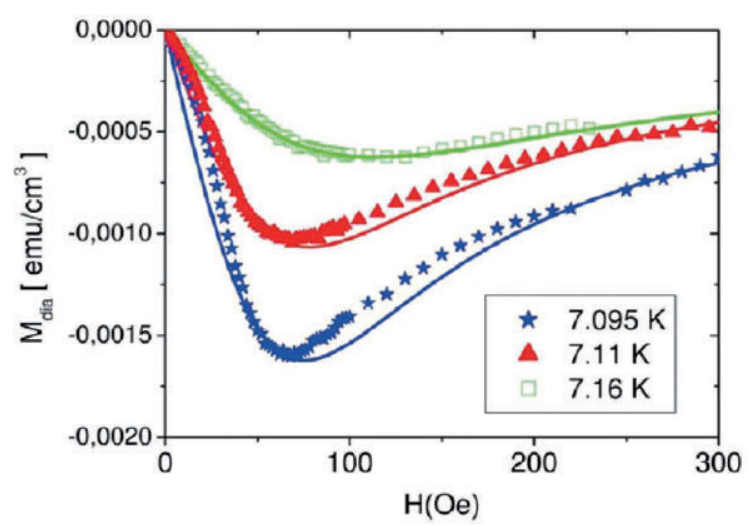

Fig. $4-\mathrm{Pb}$ nanoparticles with average diameter $d=750$ A: magnetization curves for $T$ just above $T_{c}(0)=7.09 \mathrm{~K}$. The "upturn" field, clearly evidenced by experimental data, shifts toward higher values as the temperature is increased.
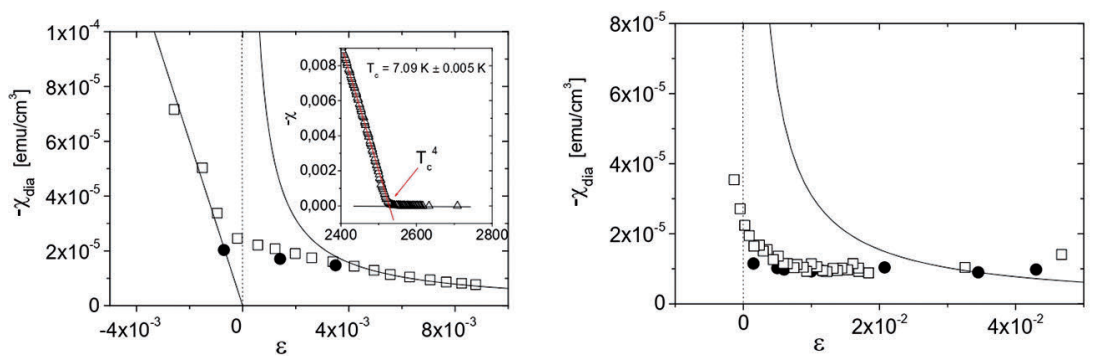

Fig. 5 - Temperature behavior of the magnetic susceptibility for Pb nanoparticles of average diameter $750 \AA$ (left) and $160 \AA$ (right) (figure from [9]). Solid circles: data from the isothermal magnetization curves; empty squares: data from the isofield measurements as a function of $T$. The solid lines report the behavior of $M_{\text {dia }}$ obtained by neglecting nonlinear fluctuations. In the inset the curve from which the transition temperature has been determined is shown ( $\varepsilon=$ reduced temperature). 


\section{High-T $\mathrm{T}_{\mathrm{C}}$ SUPERCONDUCTORS: ANOMALOUS FLUCTUATING DIAMAGNETISM}

Since 1986, a new class of high-T $\mathrm{T}_{\mathrm{c}}$ SC (HTSC) compounds has been developed. A particular ensemble of such systems is constituted by the so-called high- $\mathrm{T}_{\mathrm{c}}$ cuprates, the first example being the one of Bednorz and Muller [4], the $\mathrm{La}_{2-x} \mathrm{Ba}_{x} \mathrm{CuO}_{4}$ oxide. These systems do not follow the Bardeen-Cooper-Schrieffer (BCS) theory [3], the main coupling mechanism still being substantially unknown. The main family of high- $T_{c}$ compounds other than cuprates, are the Fe-based one [11].

In HTSC, the diamagnetic effects above $T_{c}$ depend on the charge carriers concentration $n_{h}$ [12-14]. One has to distinguish between optimally doped materials (highest $T_{c}$, optimal concentration $n_{h}{ }^{\text {opt }}$ ), underdoped compounds $\left(n_{h}<n_{h}{ }^{\text {opt }}\right)$ and overdoped ones $\left(n_{h}>n_{h}{ }^{\text {opt }}\right)$. The behaviour of $\mathrm{T}_{\mathrm{c}} v s \mathrm{n}_{\mathrm{h}}$ is plotted in Fig. 6 in order to clarify the different dependence of $T_{c}$ from the carrier concentration in the two branches.

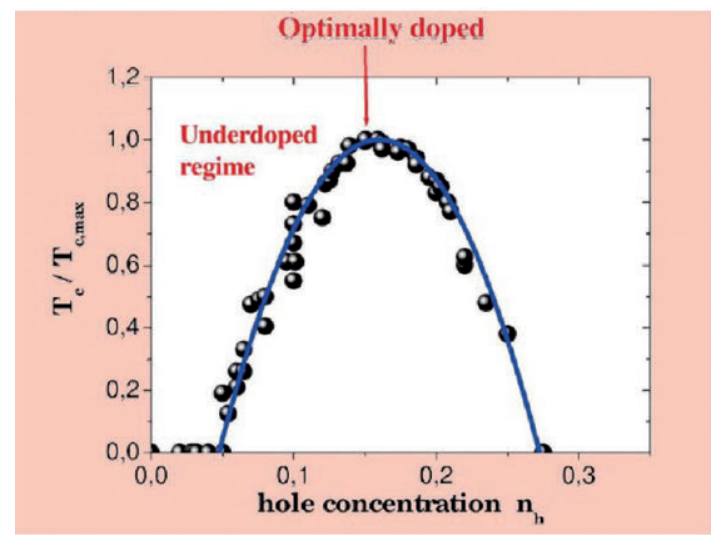

Fig. 6 - Renormalized superconducting transition temperature $T_{c}$ vs charge carriers concentration $n_{b}$. The curve is universal, for a variety of HTSC.

In the underdoped HTSC, the SF has an anomalous diamagnetic behaviour above $\mathrm{T}_{\mathrm{c}}$. The main characteristics are (see Fig. 7 for the system $\mathrm{SmBa}_{2} \mathrm{Cu}_{3-y} \mathrm{Al}_{y} \mathrm{O}_{6+\mathrm{d}}$ [15]): (i) the absolute value of the diamagnetic magnetization is particularly high, presumably due to high $\mathrm{T}_{\mathrm{c}}$ and to the short coherence length; (ii) there exists an upturn field $\mathrm{H}_{\text {up }}$ that assumes low values (with respect e.g. to the critical field ones), increases with $\mathrm{T}$ (for $\mathrm{T}>\mathrm{T}_{\mathrm{c}}$ ) and depends on the magneto-thermal history. The origin of 
this anomalous behaviour relies in the phase $(\vartheta)$ fluctuations of the order parameter, as evidenced by the model presented e.g. in Ref.s $[14,16]$. The model predicts the presence of vortexes and anti-vortexes above $T_{c}$, giving rise to mesoscopic superconducting droplets (and phase fluctuations). The irreversibility effect and the effect of the magnetic field on the thermally-activate vortexes has been predicted correctly as well. As a consequence of the existence of such phenomenon, the predicted $\mathrm{M}$ vs $\mathrm{H}$ behavior is sketched in Fig. 8, where the curves are not in scale. As seen, in addition to the observed "low" upturn field, there is a second "high" upturn field $\left(>2,5 \times 10^{4} \mathrm{Oe}\right)$ corresponding to the Cooper pairs breaking and related to the superconducting critical field.
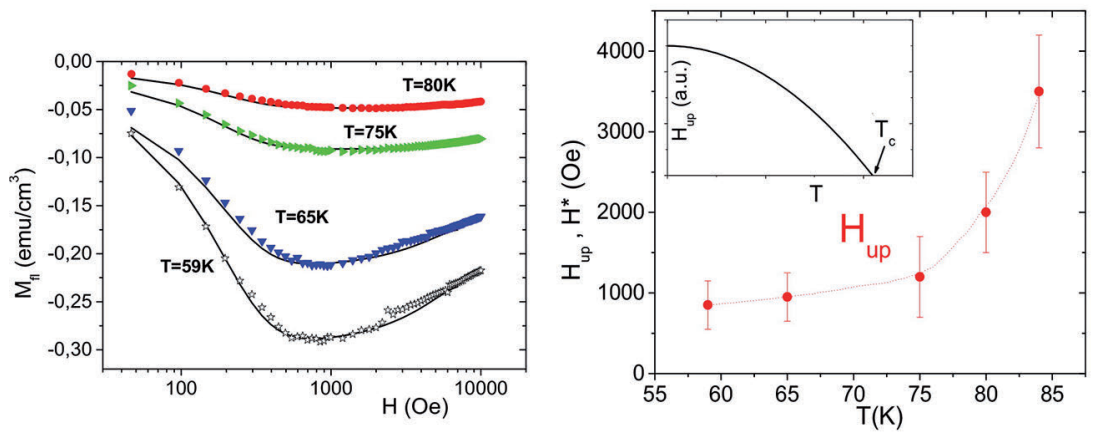

Fig. 7 - Fluctuating magnetization curves in $\mathrm{SmBa}_{2} \mathrm{Cu}_{3-y} \mathrm{Al}_{y} \mathrm{O}_{6+\delta}$ (left). The temperature behaviour of the upturn field $H_{u p}$ is reported on the right (for details on this aspect see Ref. [15]). It is noted the increase of $H_{u p}$ with T, typical of underdoped HTSC compounds.

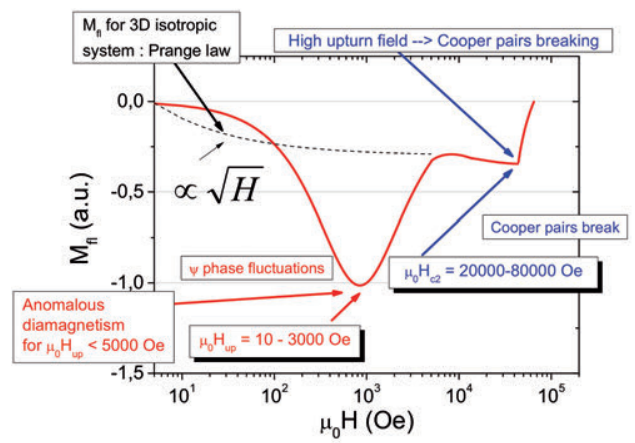

Fig. 8 - Sketchy view of the fluctuating magnetization behaviour as a function of the external field, above $T_{c}$ in a system where anomalous diamagentism occurs. At low fields, the presence of an upturn field due to phase fluctuations of the order parameter is evidenced. At high field the conventional critical field upturn due to the Cooper pairs break is shown. 


\section{SUPERCONDUCTING FLUCTUATION DIAMAGNETISM IN PNICTIDES, FE-BASED SYSTEMS AND IN CALABALACUO}

The presence of an anomalous diamagnetism of sizeable strength and of an upturn field in the isothermal fluctuating magnetization curves, has been revealed also in pnictide SC systems [17] and in Febased superconductors [18]. The fluctuating magnetization curves are shown in Fig. 9, left and right side, respectively.
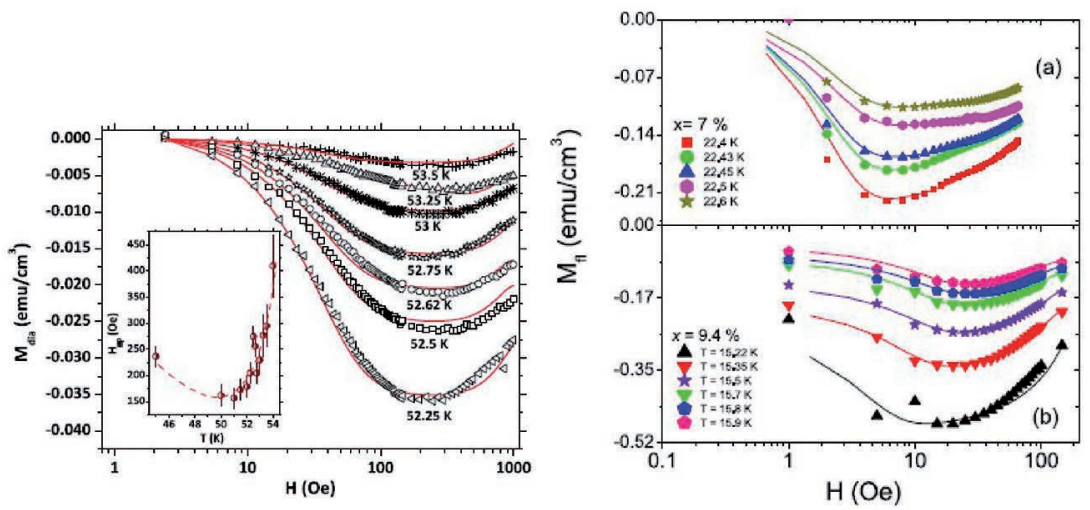

Fig. 9 - Left: isothermal fluctuating magnetization in $\mathrm{SmFeAs} \mathrm{O}_{0.8} \mathrm{~F}_{0.2}$. The upturn fields (ranging from 200 to $400 \mathrm{Oe}$ ) at different temperatures above $T^{c}\left(T_{c}=52.3 \mathrm{~K}\right.$ ) are displayed. The fitting red curves have been obtained by means of an early model reported in [14] and [15]. In the inset $H_{u p}$ behaviour, increasing by increasing $T$, is reported (dashed line is a guide to the eye). Right: isothermal fluctuating magnetization in $\mathrm{Ba}\left(\mathrm{Fe}_{1-x} \mathrm{R} \mathrm{h}_{x}\right)_{2} A s_{2}$ iron-based SC, for $x=7 \%$ and $x=9 \%$. The solid lines are fits obtained using the model in Ref. [15].

A final example concerns a particular Ca-doped compound, $\mathrm{Ca}_{x} \mathrm{La}_{1-\mathrm{x}} \mathrm{Ba}_{1.75-\mathrm{x}} \mathrm{La}_{1.25+\mathrm{x}} \mathrm{Cu}_{3} \mathrm{O}_{6+\mathrm{y}}$ (in brief CALABALACUO, first synthesized in 1993), whose peculiarity is to have the possibility of double doping (both $\mathrm{Ba} / \mathrm{Ca}$ and/or oxygen content). The crystal structure of the system is reported in Fig. 10a, while Fig. $10 b$ reports the $\mathrm{M}$ vs $\mathrm{H}$ fluctuating curves typical of an underdoped compound $(\mathrm{x}=0.4, \mathrm{y}=1.15)$ for $\mathrm{T}>\mathrm{T}_{\mathrm{c}}$. 

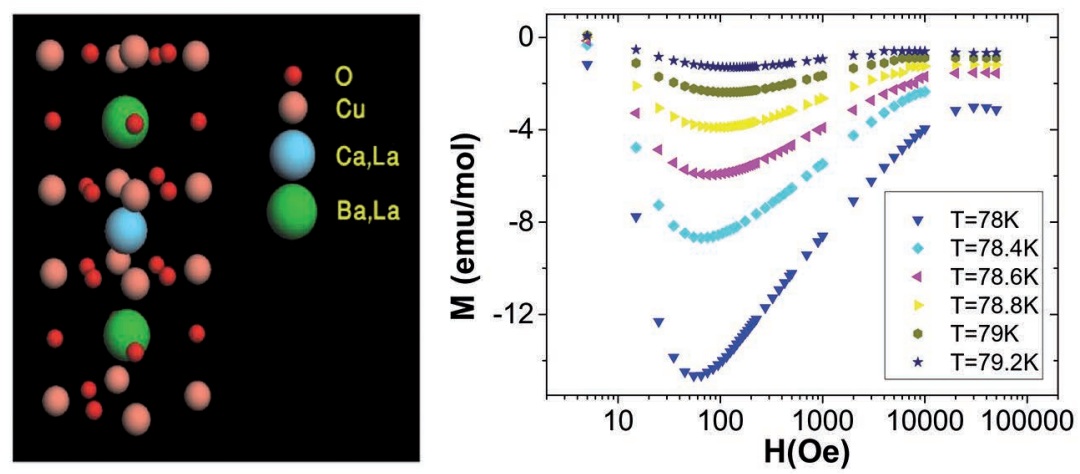

Fig. 10 - Left: Crystal structure of $\mathrm{Ca}_{x} \mathrm{La}_{1-x} \mathrm{Ba}_{1.75-x} \mathrm{La}_{1.25+x} \mathrm{Cu}_{3} \mathrm{O}_{6+y}$. Right: diamagnetic magnetization for the compound with $x=0.4, y=1.15$ (near to optimal doping but still underdoped). Even for this compounds, an upturn field is displayed for $T>T_{c}\left(T_{c}=78.4 \mathrm{~K}\right)$.

\section{CONCLUSIONS}

It has been recalled how in underdoped HTSC compounds an anomalous diamagnetism was revealed by the experimental $\mathrm{M}$ vs $\mathrm{H}$ curves collected at constant temperature just above $\mathrm{T}_{\mathrm{c}}$ (once corrected by Pauli paramagnetism and paramagnetic impurities contribution, thus giving $\mathrm{M}_{\mathrm{dia}}$, the diamagnetism fluctuating magnetization). The anomalous diamagentism is accompanied by irreversibility effects (field-cooled, FC, and zero-field-cooled, ZFC, curves different also above $\mathrm{T}_{\mathrm{c}}$ ) and can be explained in terms of mesoscopic charge inhomogeneities and of the simultaneous presence of an XY anisotropic liquid of vortexes, giving rise to phase fluctuations of the order parameter. An upturn field $\mathrm{H}_{\mathrm{up}}$ that increases with $\mathrm{T}$ above $\mathrm{T}_{\mathrm{c}}$ is the signature of such fluctuating diamagnetism, whereas for chemical inhogeneities the $\mathrm{T}$ behaviour of the upturn is demonstrated to have the opposite T-behaviour. The effect of the field is qualitatively described by the 0-D model derived from the classical GL theory: above $T_{c}$ evanescent droplets set in and an upturn field increasing with $\mathrm{T}$ is singled out. On the other hand, in optimal SC $\mathrm{M}_{\text {dia }}$ follows the conventional GL theory thus giving $\mathrm{M}_{\mathrm{dia}}$ linear in $\mathrm{H}$ for $\mathrm{T} \gg \mathrm{T}_{c}$, and $\mathrm{M}_{\mathrm{dia}} \propto \mathrm{H}^{1 / 2}$ around $\mathrm{T}_{\mathrm{c}}$.

Finally one should note that the diamagnetic behaviour could be linked to the superconducting mechanism and eventually to the pseudo-gap formation, a strongly debated issue [see e.g. 19-21]. 
From the nature of SF, the origin of the SC mechanism could be ideally deduced and it is currently under further theoretical investigation.

\section{ACKNOWLEDGMENTS}

The authors acknowledge Samuele Sanna for having allowed the publication of CALABALACUO data, and P.Battistoni and G. Prando for CALABALACUO data analysis. A. Varlamov and L. Romanò are gratefully thanked for their contribution in the theoretical analysis of the experimental data.

\section{REFERENCES}

[1] "The Feynman lectures on Physics", R. Feynman, Chapter 21, vol III, ed. Addison Wesley (1965).

[2] "Structure of Matter", $3^{\text {rd }}$ ed., A. Rigamonti and P. Carretta, ed. Springer Verlag (2015)].

[3] "Introduction to superconductivity" $2^{\text {nd }}$ ed., M. Tinkham, ed. Dover publications (2004).

[4] J.G. Bednorz and K.A. Müller, Z. Phys. B 64, 189 (1986).

[5] A.I. Larkin and A.A. Varlamov, Theory of Fluctuations in Superconductors, Clarendon, Oxford, 2005.

[6] R.E. Prange, Phys. Rev. B1, 2349 (1970).

[7] J.P. Gollub, M.R. Beasley, and M. Tinkham, Phys. Rev. Letters 25, 1646 (1970).

[8] J.P. Gollub, M.R. Beasley, R.S. Newbower, and M. Tinkham, Phys. Rev. Letters 22, 1288 (1969).

[9] E. Bernardi, A. Lascialfari, A. Rigamonti, L. Romanò, V. Iannotti, G. Ausanio, C. Luponio, Phys. Rev. B 74, 134509 (2006).

[10] A. Lascialfari, T. Mishonov, A. Rigamonti, P. Tedesco, and A.A. Varlamov, Phys. Rev. B 65, 180501R (2002).

[11] Y. Kamihara, T. Watanabe, M. Hirano, and H. Hosono, J. Am. Chem. Soc. 130, 3296 (2008).

[12] C. Baraduc, A. Buzdin, J.Y. Henry, J.P. Brison, and L. Puech, Physica C 248, 138 (1995).

[13] A. Junod, J.-Y. Genoud, G. Triscone, and T. Schneider, Physica C 294, 115 (1998).

[14] A. Lascialfari, A. Rigamonti, L. Romanò, P. Tedesco, A. Varlamov and D. Embriaco, Phys. Rev. B 65, 144523 (2002).

[15] E. Bernardi, A. Lascialfari, A. Rigamonti, L. Romanò, M. Scavini, and C. Oliva, Phys. Rev. B 81, 064502 (2010).

[16] A. Lascialfari, A. Rigamonti, L. Romano', A.A. Varlamov, I. Zucca, Phys. Rev. B 68, 100505 (R) (2003). 
[17] G. Prando et al., Phys. Rev. B 84, 064507 (2011).

[18] L. Bossoni et al., J.Phys.: Cond. Matter 26, 405703 (2014).

[19] Y. Wang, L. Li, and N.P. Ong, Phys. Rev. B 73, 024510 (2006).

[20] R.I. Rey, A. Ramos-Álvarez, J. Mosqueira, M.V. Ramallo, and F. Vidal, Phys. Rev. B 87, 056501 (2013).

[21] L. Li, Y. Wang, S. Komiya, Shimpei Ono, Y. Ando, G.D. Gu, and N.P. Ong, Phys. Rev. B 81, 054510 (2010). 
